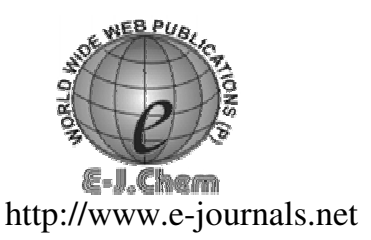

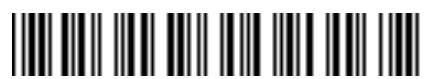

ISSN: 0973-4945; CODEN ECJHAO

E-Journal of Chemistry

Vol. 1, No. 1, pp 17-31, March 2004

\title{
Synthesis and QSAR Study of Some HDL Cholesterol Increasing Quinazolinone Derivatives
}

\author{
M.B.DESHMUKH* and SAVITA DHONGADE (DESAI). \\ Department of Chemistry, \\ Shivaji University, Kolhapur- 416004, India.
}

Received 13 February 2004; Accepted 24 February 2004

\begin{abstract}
We describe here an easy and efficient method to obtain $s$-alkylated derivatives of thio-quinazolinone using different alkylating agents via a solvent-free microwave-assisted method. The alkylated thio quinazolinones were further sequentially condensed with hydrazine hydrate and different aromatic aldehydes to get the hydrazones, which were studied for QSAR. The synthesized compounds were subjected to a prediction of biological activities. A software application (PASS) was used for this purpose. The relationship between structure and different biological activities was studied and the different derivatives were recommended for the screening of some specific activities like anti-tuberculosic, anti-mycobacterial and HDL cholesterol increasing activities.
\end{abstract}

Keywords: Synthesis, QSAR study, Thio-quinazolinone

\section{Introduction}

In recent years the use of microwave irradiation in organic reactions is rapidly increasing because of the short reaction time, operational simplicity and formation of clean reaction products. Avoiding use of organic solvent during the reactions in organic synthesis leads to a clean, efficient and economical technology (green chemistry). It has been commonly employed as thermal energy source in various organic reactions ${ }^{1}$. The use of domestic microwave oven in this regard is now a well-established procedure in $\mathrm{MORE}^{2}$ (microwave induced organic reaction enhancement) chemistry. It has been reported that the rate of variety of organic reactions such as Diels-Alder ${ }^{3}$, ene ${ }^{4}$, Claisen reaction ${ }^{5}$, oxidation ${ }^{6}$, reduction $^{7}$, diacetylation ${ }^{8}$, deacetylation ${ }^{9}$, deoximation ${ }^{10}$, esterification ${ }^{11}$, hydrolysis of 
ester $^{12}$, Doebner condensation ${ }^{13}$, Knoevenagel condensation ${ }^{14}$ coulds be enhanced by microwave irradiation. Several workers have reported the alkylation of $N$-containing heterocycles. In this regard microwave (MW) activation have been successfully applied in the synthesis of such derivatives ${ }^{15-17}$.

Quinazoline derivatives are of special importance because of their versatile biological \& pharmacological activities ${ }^{18-20}$, especially anti-inflammatory ${ }^{21-23}$, anticonvulsant $^{24}$, hypnotic $^{25}$, anthemintic ${ }^{26}$, hypo-tensive ${ }^{27}$, antibacterial ${ }^{28}$ agents etc. In the present work, $s$-alkylated derivatives of thio-quinazolinone were obtained using Ethyl chloroacetate via a solvent-free microwave-assisted method. The alkylated thio quinazolinones were further sequentially condensed with hydrazine hydrate and different aromatic aldehydes to get the hydrazides, which were studied for QSAR. The synthesized compounds were subjected to a prediction of biological activities. A software application (PASS $)^{29}$ was used for this purpose. The relationship between structure and different biological activities was studied and the different derivatives were recommended for the screening of some specific activities.

\section{Experimental}

All m.ps. were determined in open capillary tubes and are uncorrected. IR spectra were recorded in $\mathrm{KBr}$ on Shimadzu IR-437 spectrophotometer and PMR spectra in $\mathrm{CDCl}_{3}$ and DMSO- $\mathrm{d}_{6}$ on Perkin-Elmer R-32 spectrometer using TMS as an internal standard. The purity of the compounds was checked by TLC. Microwave irradiation was carried out in the domestic microwave oven by SHARP.

\section{2-Mercapto-3-o-tolyl-3H-quinazolin-4-one (I a)}

2-Mercapto-3-o-tolyl-3H-quinazolin-4-one ( $\mathrm{I}$ a) was synthesized by a reported $\operatorname{method}^{30}$ as follows.

The mixture of 2-methyl aniline $(0.1$ mole) was dissolved in benzene $(12 \mathrm{~mL})$ and carbon disulphide (11.7 mL, 0.15 mole) and triethylamine (15.45 g., 0.15 mole) stirred mechanically at $0^{0} \mathrm{C}$ to get triethylammonium dithiocarbamate salt. The salt was then filtered, washed with dry ether, dried and crystallized from chloroform to get dithiocarbamate salts. Further the mixture of triethylammonium $\mathrm{N}$ - (2-methylphenyl) dithiocarbamate (0.05 mole) and anthranilic acid ( $7.2 \mathrm{~g} ., 0.05$ mole) in ethanol $(25 \mathrm{~mL})$ was refluxed on a steam bath for $6 \mathrm{~h}$, cooled and the separated solid was filtered and washed with water and further dissolved in $10 \%$ ethanolic sodium hydroxide solution, filtered and reprecipitated by the addition of dilute hydrochloric acid. The product obtained was filtered, washed with water and recrystallized from ethanol to give Ia. Yield: $89 \%$, M.P. $291^{\circ} \mathrm{C}$., Mol.Formula : $\mathrm{C}_{15} \mathrm{H}_{12} \mathrm{~N}_{2} \mathrm{OS}$, Elemental analysis: C, 67.0(67.14 \%) ; H, 4.5(4.51\%) ; N, 10.5(10.44\%), IR(KBr): vmax, 3320-

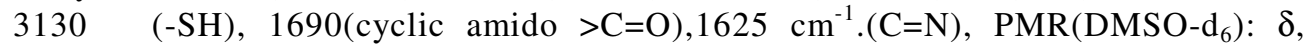
$2.9\left(3 \mathrm{H}, \mathrm{s}, \mathrm{Ar}-\mathrm{CH}_{3}\right), \quad 7.2-8.1(8 \mathrm{H}, \mathrm{m}, \mathrm{Ar}-\mathrm{H}), \quad 10.8\left(1 \mathrm{H}, \mathrm{s}\right.$, exchangeable with $\left.\mathrm{D}_{2} \mathrm{O},-\mathrm{SH}\right)$ ppm.

Similarly, 2-Mercapto-3-m-tolyl-3H-quinazolin-4-one (I b), 2-Mercapto-3-ptolyl-3H-quinazolin-4-one (I c), 3-(3-Chloro-phenyl)-2-mercapto-3H-quinazolin-4one (I d),3-(4-Chloro-phenyl) -2- mercapto -3H - quinazolin -4-one (I e), 2 Mercapto - 3- (3-methoxy-phenyl) -3H -quinazolin-4-one (I f) and 2-Mercapto-(4methoxy-phenyl) -3H-quinazolin-4-one (I g) were synthesized. 


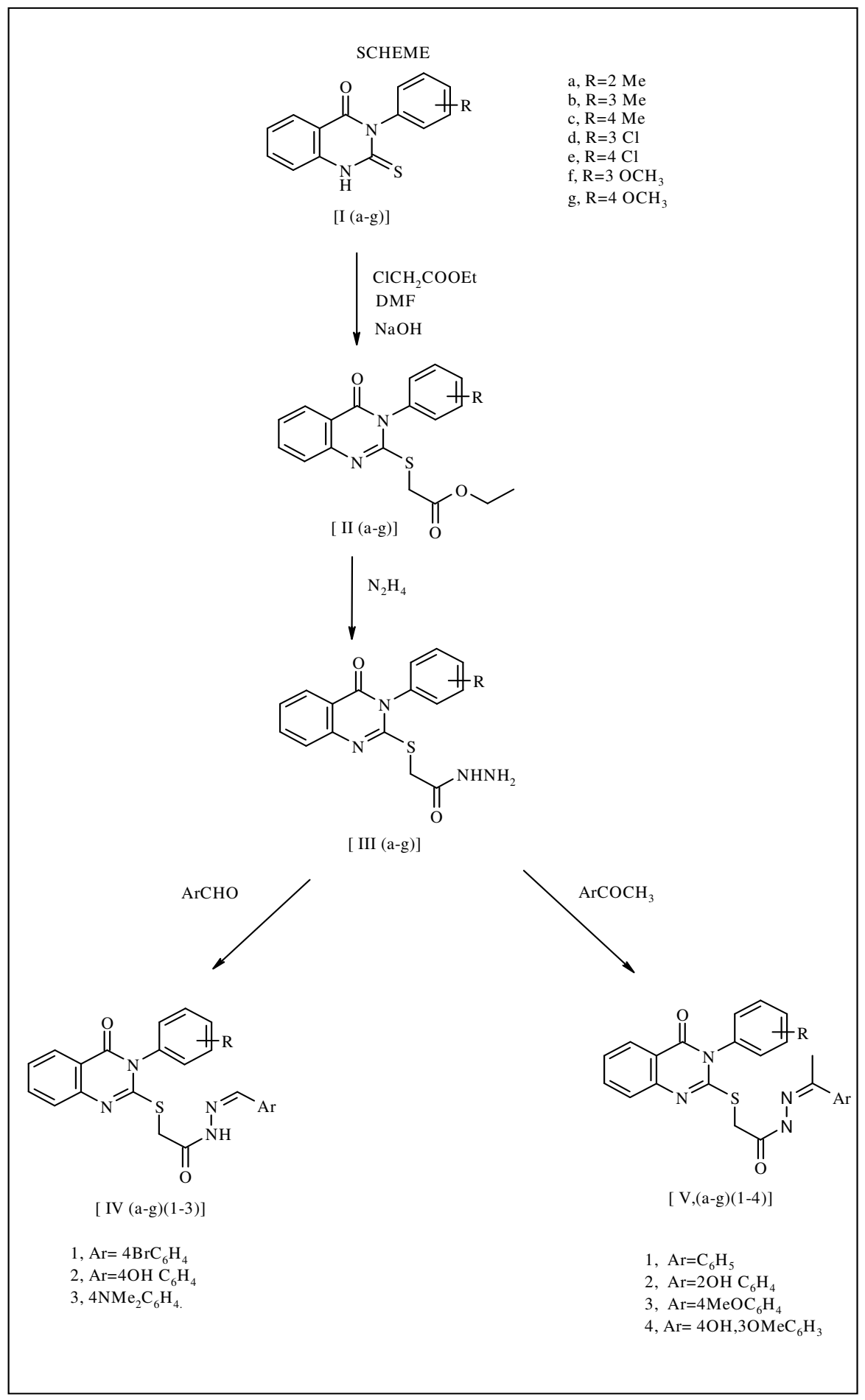


Table 1. General characteristics and elemental analysis data of the compounds I (a-g)

\begin{tabular}{|c|c|c|c|c|c|c|}
\hline Compound & $\begin{array}{l}\text { Yield } \\
\%\end{array}$ & $\begin{array}{l}\mathrm{M} . \mathrm{P} \\
{ }^{\circ} \mathrm{C}\end{array}$ & $\begin{array}{c}\text { Mol. } \\
\text { Formula }\end{array}$ & $\begin{array}{c}\text { Carbon, \% } \\
\text { (found calcd) }\end{array}$ & $\begin{array}{l}\text { Hydrogen, \% } \\
\text { (found calcd) }\end{array}$ & $\begin{array}{l}\text { Nitrogen, \% } \\
\text { (found calcd) }\end{array}$ \\
\hline \multirow{2}{*}{ I a } & \multirow{2}{*}{79} & \multirow{2}{*}{256} & \multirow{2}{*}{$\mathrm{C}_{15} \mathrm{H}_{12} \mathrm{~N}_{2} \mathrm{OS}$} & 67.0 & 4.5 & 10.5 \\
\hline & & & & 67.14 & 4.51 & 10.44 \\
\hline \multirow{2}{*}{$\mathrm{I} \mathrm{b}$} & \multirow{2}{*}{89} & \multirow{2}{*}{268} & \multirow{2}{*}{$\mathrm{C}_{15} \mathrm{H}_{12} \mathrm{~N}_{2} \mathrm{OS}$} & 67.10 & 4.54 & 10.45 \\
\hline & & & & 67.14 & 4.51 & 10.44 \\
\hline \multirow{2}{*}{ I c } & \multirow{2}{*}{98} & \multirow{2}{*}{302} & \multirow{2}{*}{$\mathrm{C}_{15} \mathrm{H}_{12} \mathrm{~N}_{2} \mathrm{OS}$} & 67.0 & 4.45 & 10.40 \\
\hline & & & & 67.14 & 4.51 & 10.44 \\
\hline \multirow{2}{*}{ I d } & \multirow{2}{*}{85} & \multirow{2}{*}{270} & $\mathrm{C}_{14} \mathrm{H}_{9} \mathrm{ClN}_{2} \mathrm{O}$ & 58.2 & 3.1 & 9.8 \\
\hline & & & S & 58.23 & 3.14 & 9.70 \\
\hline \multirow{2}{*}{$\mathrm{I} \mathrm{e}$} & \multirow{2}{*}{84} & \multirow{2}{*}{315} & $\mathrm{C}_{14} \mathrm{H}_{9} \mathrm{ClN}_{2} \mathrm{O}$ & 58.3 & 3.2 & 9.75 \\
\hline & & & S & 58.23 & 3.14 & 9.70 \\
\hline \multirow{2}{*}{ If } & \multirow{2}{*}{86} & \multirow{2}{*}{280} & $\mathrm{C}_{15} \mathrm{H}_{12} \mathrm{~N}_{2} \mathrm{O}_{2}$ & 63.3 & 4.2 & 9.8 \\
\hline & & & S & 63.36 & 4.25 & 9.85 \\
\hline \multirow{2}{*}{ I g } & \multirow{2}{*}{78} & \multirow{2}{*}{273} & $\mathrm{C}_{15} \mathrm{H}_{12} \mathrm{~N}_{2} \mathrm{O}_{2}$ & 63.3 & 4.2 & 9.8 \\
\hline & & & S & 63.36 & 4.25 & 9.85 \\
\hline
\end{tabular}

Table 2.Spectral Characteristics

\begin{tabular}{lll}
\hline Comp & IR vmax, $\mathrm{cm}^{-1}$ & PMR $\delta$, ppm. \\
\hline Ib & $3380-3200(-\mathrm{SH}), 1680-1670$ & $3.80\left(3 \mathrm{H}, \mathrm{s}, \mathrm{CH}_{3}\right)$, \\
& (cyclic amido $>\mathrm{C}=\mathrm{O})$, & $6.9-7.9(8 \mathrm{H}, \mathrm{m}, \mathrm{Ar}-\mathrm{H}), 11.9(1 \mathrm{H}, \mathrm{s}, \mathrm{Br}$. \\
& $1625(\mathrm{C}=\mathrm{N})$, & Exchangeable with $\left.\mathrm{D}_{2} \mathrm{O}, \mathrm{NH}\right)$ \\
Ic & $3150-3200(-\mathrm{NH}), 1680$ & $2.3\left(3 \mathrm{H}, \mathrm{s}, \mathrm{CH}_{3}\right)$, \\
& $($ cyclic amido $>\mathrm{C}=\mathrm{O})$, & $7.8-7.9(8 \mathrm{H}, \mathrm{m}, \mathrm{Ar}-\mathrm{H}), 11.3(1 \mathrm{H}, \mathrm{s}, \mathrm{br}$. \\
& $1620(\mathrm{C}=\mathrm{N})$, & Exchangeablewith $\left.\mathrm{D}_{2} \mathrm{O}, \mathrm{NH}\right)$ \\
Id & $3325-3135(-\mathrm{NH}), 1690$ & $7.2-8.1(8 \mathrm{H}, \mathrm{m}, \mathrm{Ar}-\mathrm{H})$, \\
& $($ cyclic amido $>\mathrm{C}=\mathrm{O})$, & $12.8(1 \mathrm{H}, \mathrm{s}, \mathrm{br}$, exchangeable \\
& $1625(\mathrm{C}=\mathrm{N}), 760(\mathrm{C}-\mathrm{Cl})$ & with $\left.\mathrm{D}_{2} \mathrm{O}, \mathrm{NH}\right)$ \\
Ie & $3150-3200(-\mathrm{NH}), 1680$ & $7.5-8.3(8 \mathrm{H}, \mathrm{m}, \mathrm{Ar}-\mathrm{H}), 12.8(1 \mathrm{H}, \mathrm{s}, \mathrm{br}$, \\
& $($ cyclic amido $>\mathrm{C}=\mathrm{O})$, & exchangeable with $\left.\mathrm{D}_{2} \mathrm{O}, \mathrm{NH}\right)$ \\
& $1620(\mathrm{C}=\mathrm{N}), 760(\mathrm{C}-\mathrm{Cl})$ & \\
If & $3250-3125(-\mathrm{NH}), 1675-1680$ & $3.7\left(3 \mathrm{H}, \mathrm{s}, \mathrm{OCH} \mathrm{CH}_{3}\right), 6.7-$ \\
& $($ cyclic amido $>\mathrm{C}=\mathrm{O})$, & $8.15(8 \mathrm{H}, \mathrm{m}, \mathrm{Ar}-\mathrm{H}), 12.9(1 \mathrm{H}, \mathrm{s}, \mathrm{br}$, \\
& $1620(\mathrm{C}=\mathrm{N}), 1060(-\mathrm{O}-)$ & exchangeable with $\left.\mathrm{D}_{2} \mathrm{O}, \mathrm{NH}\right)$ \\
Ig & $3315-3125(-\mathrm{NH}), 1680$ & $\left.3.8(3 \mathrm{H}, \mathrm{s}, \mathrm{OCH})_{3}\right)$, \\
& $($ cyclic amido $>\mathrm{C}=\mathrm{O})$, & $6.8-8.25(8 \mathrm{H}, \mathrm{m}, \mathrm{Ar}-\mathrm{H}), 13.0(1 \mathrm{H}, \mathrm{s}, \mathrm{br}$, \\
& $1625(\mathrm{C}=\mathrm{N}),(-\mathrm{O}-)$ & exchangeable with $\left.\mathrm{D}_{2} \mathrm{O}, \mathrm{NH}\right)$ \\
\hline
\end{tabular}

[4-Oxo-3-o-tolyl-3,4-dihydro-quinazolin-2-ylsulfanyl]-acetic acid ethyl ester [II a]

$2.5 \mathrm{mmol}$ of (Ia), (2.5) mmol of ethyl chloroacetate and $1 \mathrm{mmol}$ of DMF and powdered $\mathrm{NaOH}(0.5 \mathrm{~g})$, were smoothly mixed and placed inside a Pyrex-glass open vessel .The mixture was irradiated with microwaves ( $900 \mathrm{~W} / 2450 \mathrm{MHz}$ frequency) for the specified time on $50 \%$ power using a domestic microwave oven 'Sharp R-758B'. When the irradiation was 
stopped, the final temperature was measured by introducing a glass thermometer into the homogenized reaction mixture. The mixture was added with $2 \mathrm{~N} \mathrm{HCl}(10 \mathrm{~mL})$. The solid separated was filtered, conveniently dried and recrystallized of water to get $\mathrm{IIa}_{1}$. Yield $79 \%$, M.P. $142^{\circ} \mathrm{C}$, Molecular formula $\mathrm{C}_{19} \mathrm{H}_{18} \mathrm{~N}_{2} \mathrm{O}_{3} \mathrm{~S}$, elemental analysis $\mathrm{C}, 64.5$ (64.41); H, 5.0 (5.08); N, 7.8 (7.91). IR(KBr): vmax, $1750(\mathrm{C}=\mathrm{O}), 1710($ cyclic $\mathrm{C}=\mathrm{O}), 1620 \mathrm{~cm}^{-1}(\mathrm{C}=\mathrm{N})$, PMR(DMSO-d $\left.\mathrm{d}_{6}\right): \delta, 1.3\left(3 \mathrm{H}, \mathrm{t} \mathrm{J}=7 \mathrm{~Hz} ., \mathrm{CH}_{3}\right), 2.9\left(3 \mathrm{H}, \mathrm{s}, \mathrm{Ar}-\mathrm{CH}_{3}\right) 3.64\left(2 \mathrm{H}, \mathrm{s},-\mathrm{SCH}_{2}\right), \quad 4.3(2 \mathrm{H}, \mathrm{q}$ $\left.\mathrm{J}=7 \mathrm{~Hz} ., \mathrm{CH}_{2}\right), 7.5-8.2(8 \mathrm{H}, \mathrm{m}, \mathrm{Ar}-\mathrm{H}) \mathrm{ppm}$.

Similarly,[4-Oxo-3-m-tolyl-3,4-dihydro-quinazolin-2-ylsulfanyl]-acetic acid ethyl ester (II b), [4-Oxo-3-p-tolyl-3,4-dihydro-quinazolin-2-ylsulfanyl]-acetic acid ethyl ester (II c), [3-(3-Chloro-phenyl)-4-oxo-3,4-dihydro-quinazolin-2-ylsulfanyl]acetic acid ethyl ester (II d), [3-(4-Chloro-phenyl)-4-oxo-3,4-dihydro-quinazolin-2-ylsulfanyl] acetic acid ethyl ester (II e), ),[3-(3-methoxy-phenyl)-4-oxo-3,4-dihydro-quinazolin-2-ylsulfanyl]acetic acid ethyl ester (II f) and [3-(4-methoxy-phenyl)-4-oxo-3,4-dihydro-quinazolin-2ylsulfanyl]acetic acid ethyl ester (II g) were synthesized .Their structures were confirmed on the basis of elemental and spectral characteristics.

Table 3. General characteristics and elemental analysis data of the compounds II (a-g)

\begin{tabular}{|c|c|c|c|c|c|c|}
\hline Compound & $\begin{array}{c}\text { Yield } \\
\%\end{array}$ & $\underset{{ }^{\circ} \mathrm{C}}{\mathrm{M} . \mathrm{P}}$ & Mol. Formula & $\begin{array}{l}\text { Carbon } \\
\% \text { (found } \\
\text { calcd) }\end{array}$ & $\begin{array}{l}\text { Hydrogen } \\
\% \text { (found } \\
\text { calcd) }\end{array}$ & $\begin{array}{c}\text { Nitrogen } \\
\% \text { (found } \\
\text { calcd) }\end{array}$ \\
\hline IIa & 79 & 142 & $\mathrm{C}_{19} \mathrm{H}_{18} \mathrm{~N}_{2} \mathrm{O}_{3} \mathrm{~S}$ & $\begin{array}{c}64.4 \\
64.39\end{array}$ & $\begin{array}{c}5.1 \\
5.12\end{array}$ & $\begin{array}{l}7.85 \\
7.90\end{array}$ \\
\hline $\mathrm{IIb}$ & 86 & 123 & $\mathrm{C}_{19} \mathrm{H}_{18} \mathrm{~N}_{2} \mathrm{O}_{3} \mathrm{~S}$ & $\begin{array}{l}64.35 \\
64.39\end{array}$ & $\begin{array}{l}5.11 \\
5.12\end{array}$ & $\begin{array}{l}7.92 \\
7.90\end{array}$ \\
\hline IIc & 89 & 102 & $\mathrm{C}_{19} \mathrm{H}_{18} \mathrm{~N}_{2} \mathrm{O}_{3} \mathrm{~S}$ & $\begin{array}{l}64.42 \\
64.39\end{array}$ & $\begin{array}{l}5.20 \\
5.12\end{array}$ & $\begin{array}{l}7.91 \\
7.90\end{array}$ \\
\hline IId & 84.5 & 86 & $\mathrm{C}_{18} \mathrm{H}_{15} \mathrm{ClN}_{2} \mathrm{O}_{3} \mathrm{~S}$ & $\begin{array}{c}57.5 \\
57.68\end{array}$ & $\begin{array}{l}4.00 \\
4.03\end{array}$ & $\begin{array}{c}7.4 \\
7.47\end{array}$ \\
\hline IIe & 89 & 98 & $\mathrm{C}_{18} \mathrm{H}_{15} \mathrm{ClN}_{2} \mathrm{O}_{3} \mathrm{~S}$ & $\begin{array}{l}57.59 \\
57.68\end{array}$ & $\begin{array}{l}4.10 \\
4.03\end{array}$ & $\begin{array}{l}7.45 \\
7.47\end{array}$ \\
\hline IIf & 79 & 126 & $\mathrm{C}_{19} \mathrm{H}_{18} \mathrm{~N}_{2} \mathrm{O}_{4} \mathrm{~S}$ & $\begin{array}{c}61.6 \\
61.61\end{array}$ & $\begin{array}{l}4.89 \\
4.90\end{array}$ & $\begin{array}{l}7.50 \\
7.56\end{array}$ \\
\hline $\mathrm{IIg}$ & 85 & 122 & $\mathrm{C}_{19} \mathrm{H}_{18} \mathrm{~N}_{2} \mathrm{O}_{4} \mathrm{~S}$ & $\begin{array}{l}61.56 \\
61.61\end{array}$ & $\begin{array}{l}4.85 \\
4.90\end{array}$ & $\begin{array}{l}7.53 \\
7.56\end{array}$ \\
\hline
\end{tabular}

(4-Oxo-3-o-tolyl-3,4-dihydro-quinazolin-2-ylsulfanyl)-acetic acid hydrazide(III a)

IIa $(0.1$ mole) was mixed with 0.1 mole of hydrazine hydrate in $5 \mathrm{~mL}$ of methanol and refluxed on a steam bath for three hours. The separated solid was filtered and recrystalized from ethanol to get IIIa. Yield $84 \%$, M.P. $185^{\circ} \mathrm{C}$, Mol. Wt.= 340,Mol. Formula $: \mathrm{C}_{17} \mathrm{H}_{16} \mathrm{~N}_{4} \mathrm{O}_{2} \mathrm{~S}$, Elemental Analysis:C,59.9(59.98)\%); H,4.70(4.74\%); 16.99(16.96\%), IR (KBr): vmax, 3300-3250 $\left(\mathrm{NHNH}_{2}\right), 1680(>\mathrm{C}=\mathrm{O}), 1660(>\mathrm{C}=\mathrm{O}), 1620 \mathrm{~cm}^{-1}(\mathrm{C}=\mathrm{N}), \mathrm{PMR}\left(\mathrm{DMSO}-\mathrm{d}_{6}\right): \delta, 2.9(3 \mathrm{H}, \mathrm{s}$, $\left.\mathrm{Ar}-\mathrm{CH}_{3}\right), \quad 3.74(2 \mathrm{H}, \mathrm{s}, \mathrm{SCH} 2), 4.45\left(1 \mathrm{H}, \mathrm{q}, \mathrm{J}=8.5 \mathrm{~Hz},-4.35\left(2 \mathrm{H}, \mathrm{s}, \mathrm{NH}_{2}\right), \quad 7.4-8.2(8 \mathrm{H}, \mathrm{m}, \mathrm{Ar}-\mathrm{H})\right.$, $9.55(1 \mathrm{H}, \mathrm{s}, \mathrm{CONH}) \mathrm{ppm}$. 
Similarly, (4-Oxo-3-m-tolyl-3,4-dihydro-quinazolin-2-ylsulfanyl)-acetic acid hydrazide (III b),(4-Oxo-3-p-tolyl-3,4-dihydro-quinazolin-2-ylsulfanyl)-acetic acid hydrazide (III c), [3-(3-Chloro-phenyl)-4-oxo-3,4-dihydro-quinazolin-2-ylsulfanyl]-acetic acid hydrazide (III d), [3-(4-Chloro-phenyl)-4-oxo-3,4-dihydro-quinazolin-2-ylsulfanyl]-acetic acid hydrazide (III e), [3-(3-Methoxy -phenyl)-4-oxo-3,4-dihydro-quinazolin-2-ylsulfanyl]-acetic acid hydrazide (III f) and [3-(4-Methoxy-phenyl)-4-oxo-3,4-dihydro-quinazolin-2ylsulfanyl]-acetic acid hydrazide (III g) were synthesized.

Table 4. General characteristics and elemental analysis data of the compounds III (a-g)

\begin{tabular}{|c|c|c|c|c|c|c|}
\hline Compound & $\begin{array}{c}\text { Yield } \\
\%\end{array}$ & $\begin{array}{c}\text { M.P. } \\
{ }^{\circ} \mathrm{C}\end{array}$ & Mol. Formula & $\begin{array}{c}\text { Carbon } \\
\%, \text { (found } \\
\text { Calcd) }\end{array}$ & $\begin{array}{c}\text { Hydrogen } \\
\% \text {, (found } \\
\text { Calcd) }\end{array}$ & $\begin{array}{c}\text { Nitrogen } \\
\% \text {, (found } \\
\text { Calcd) }\end{array}$ \\
\hline \multirow{2}{*}{ IIIb } & \multirow{2}{*}{90} & \multirow{2}{*}{168} & \multirow[b]{2}{*}{$\mathrm{C}_{17} \mathrm{H}_{16} \mathrm{~N}_{4} \mathrm{O}_{2} \mathrm{~S}$} & 59.2 & 4.68 & 16.51 \\
\hline & & & & 59.98 & 4.74 & 16.46 \\
\hline \multirow{2}{*}{ IIIc } & \multirow{2}{*}{86} & \multirow{2}{*}{148} & \multirow{2}{*}{$\mathrm{C}_{17} \mathrm{H}_{16} \mathrm{~N}_{4} \mathrm{O}_{2} \mathrm{~S}$} & 60.0 & 4.75 & 16.45 \\
\hline & & & & 59.98 & 4.74 & 16.46 \\
\hline \multirow{2}{*}{ IIId } & \multirow{2}{*}{87} & \multirow{2}{*}{186} & \multirow{2}{*}{$\mathrm{C}_{16} \mathrm{H}_{13} \mathrm{ClN}_{4} \mathrm{O}_{2} \mathrm{~S}$} & 53.2 & 3.6 & 15.5 \\
\hline & & & & 53.26 & 3.63 & 15.53 \\
\hline \multirow{2}{*}{ IIIe } & \multirow{2}{*}{84} & \multirow{2}{*}{175} & \multirow{2}{*}{$\mathrm{C}_{16} \mathrm{H}_{13} \mathrm{ClN}_{4} \mathrm{O}_{2} \mathrm{~S}$} & 53.25 & 3.61 & 15.55 \\
\hline & & & & 53.26 & 3.63 & 15.53 \\
\hline \multirow{2}{*}{ IIIf } & \multirow{2}{*}{88} & \multirow{2}{*}{156} & \multirow{2}{*}{$\mathrm{C}_{17} \mathrm{H}_{16} \mathrm{~N}_{4} \mathrm{O}_{3} \mathrm{~S}$} & 57.2 & 4.5 & 15.8 \\
\hline & & & & 57.29 & 4.53 & 15.72 \\
\hline \multirow{2}{*}{ IIIg } & \multirow{2}{*}{89} & \multirow{2}{*}{149} & \multirow{2}{*}{$\mathrm{C}_{17} \mathrm{H}_{16} \mathrm{~N}_{4} \mathrm{O}_{3} \mathrm{~S}$} & 57.27 & 4.51 & 15.71 \\
\hline & & & & 57.29 & 4.53 & 15.72 \\
\hline
\end{tabular}

(4-Oxo-3-o-tolyl-3,4-dihydro-quinazolin-2-ylsulfanyl)-acetic acid (4-bromobenzyle -dene)hydrazide (IV a-1)

0.1 mole of III a and 0.1 mole of 4-bromobenzaldehyde, $5 \mathrm{~mL}$ of DMF and few drops of acetic acid were smoothly mixed and placed inside a Pyrex-glass Erlenmeyer flask loosely corked with cotton. The mixture was irradiated with microwaves $(900 \mathrm{~W} / 2450 \mathrm{MHz}$ frequency) for 5 minutes on $50 \%$ power using a domestic microwave oven 'Sharp R-758B'. When the irradiation was stopped, the mixture was added to ice-water mixture. The solid separated was filtered, conveniently dried and recrystallized of water to get IVa. Yield 88\%, M.P. $322^{\circ} \mathrm{C}$., Mol. Formula : $\mathrm{C}_{24} \mathrm{H}_{19} \mathrm{BrN}_{4} \mathrm{O}_{2} \mathrm{~S}$, Elemental analysis: Carbon, 56.8(56.81\%); Hydrogen, 3.7(3.77\%); Nitrogen, 11.0(11.04 \%) ,IR (KBr): vmax, 1720(cyclic amido), 1680(acyclic >C=O), $1610 \mathrm{~cm}^{-1} .(\mathrm{C}=\mathrm{N}), \quad \mathrm{PMR}\left(\mathrm{DMSO}-\mathrm{d}_{6}\right): \quad \delta, 2.9\left(3 \mathrm{H}, \mathrm{s}, \mathrm{Ar}_{-} \mathrm{CH}_{3}\right)$, 3.74(2H,s, $\left.\mathrm{SCH}_{2}\right), 7.3-8.3(12 \mathrm{H}, \mathrm{m}, \mathrm{Ar}-\mathrm{H}), 9.6(1 \mathrm{H}, \mathrm{s}, \mathrm{CONH}) \mathrm{ppm}$.

Similarly,(4-Oxo-3-m-tolyl-3,4-dihydro-quinazolin-2-ylsulfanyl)-acetic acid (4-bromobenzyledene)-hydrazide (IVb-1), (4-Oxo-3-p-tolyl-3,4-dihydro-quinazolin-2-ylsulfanyl)acetic acid (4-bromo-benzyledene)-hydrazide (IVc-1), (4-Oxo-3-o-tolyl-3,4-dihydroquinazolin-2-ylsulfanyl)-acetic acid (4-hydroxy -benzyledene)-hydrazide (IVa-2), (4-Oxo-3m-tolyl-3,4-dihydro-quinazolin-2-ylsulfanyl)-acetic acid (4-hydroxy-benzyledene)-hydrazide (IVb-2),(4-Oxo-3-p-tolyl-3,4-dihydro-quinazolin-2-ylsulfanyl)-acetic acid (4-hydroxy benzyledene)-hydrazide (IVc-2), (4-Oxo-3-o-tolyl-3,4-dihydro-quinazolin-2-ylsulfanyl)acetic acid (4 - dimethylamino - benzyledene) - hydrazide (IVa-3), (4-Oxo-3-m-tolyl-3,4- 
dihydro-quinazolin-2-ylsulfanyl)-acetic acid (4-dimethylamino-benzyledene)-hydrazide (IVb-3), (4-Oxo-3-p-tolyl-3,4-dihydro-quinazolin-2-ylsulfanyl)-acetic acid (4dimethylamino-benzyledene)-hydrazide (IVc-3), [3-(3-Chloro-phenyl)-4-oxo-3,4-dihydroquinazolin-2-ylsulfanyl]-acetic acid (4-bromo-benzyledene)-hydrazide (IV d-1), [3-(4Chloro-phenyl)-4-oxo-3,4-dihydro-quinazolin-2-ylsulfanyl]-acetic acid (4-bromobenzyledene)-hydrazide (IV e-1), [3-(3-Chloro-phenyl)-4-oxo-3,4-dihydro-quinazolin-2ylsulfanyl]-acetic acid (4-hydroxy-benzyledene)hydrazide (IV d-2), [3-(4-Chloro-phenyl)-4oxo-3,4-dihydro-quinazolin-2-ylsulfanyl]-acetic acid (4-hydroxy-benzyledene) hydrazide (IVe-2), [3-(3-Chloro-phenyl)-4-oxo-3,4-dihydro-quinazolin-2-ylsulfanyl]-acetic acid (4-dimethylamino- benzyledene)-hydrazide (IV d-3), [3-(4-Chloro-phenyl)-4-oxo-3,4dihydro-quinazolin-2-ylsulfanyl]-acetic acid (4-dimethylamino -benzyledene)-hydrazide (IV e-3), [3-(3-Methoxy-phenyl)-4-oxo-3,4-dihydro-quinazolin-2-ylsulfanyl]-acetic acid (4bromo-benzyledene)-hydrazide (IV f-1), [3-(4-Methoxy -phenyl)-4-oxo-3,4-dihydroquinazolin-2-ylsulfanyl]-acetic acid (4-bromo-benzyledene)-hydrazide (IV g-1), [3-(3Methoxy-phenyl)-4-oxo-3,4-dihydro-quinazolin-2-ylsulfanyl]-acetic acid (4-hydroxybenzyledene)-hydrazide (IV f-2), [3-(4-Methoxy -phenyl)-4-oxo-3,4-dihydro-quinazolin-2ylsulfanyl]-acetic acid (4-hydroxy-benzyledene)-hydrazide (IVg-2), [3-(3-Methoxy-phenyl)4-oxo-3,4-dihydro-quinazolin-2-ylsulfanyl]-acetic acid (4-dimethylamino-benzyledene)hydrazide (IVf-3), [3-(4-Methoxy-phenyl)-4-oxo-3,4-dihydro-quinazolin-2-ylsulfanyl]-acetic acid (4-dimethylamino -benzyledene)-hydrazide (IVg-3) were synthesised and subjected to PASS for prediction of biological activities.

Table 5. General characteristics and elemental analysis data of the compounds IV (a-g)(1-3)

\begin{tabular}{|c|c|c|c|c|c|c|}
\hline Compound & $\begin{array}{c}\text { Yield } \\
\%\end{array}$ & $\underset{{ }^{\circ} \mathrm{C}}{\mathrm{M} . \mathrm{P}}$ & Mol. Formula & $\begin{array}{c}\text { Carbon } \\
\%, \text { (found } \\
\text { Calcd) }\end{array}$ & $\begin{array}{c}\text { Hydrogen } \\
\% \text {, (found } \\
\text { Calcd) }\end{array}$ & $\begin{array}{c}\text { Nitrogen } \\
\%, \text { (found } \\
\text { Calcd) }\end{array}$ \\
\hline IV a-1 & 88 & 322 & $\mathrm{C}_{24} \mathrm{H}_{19} \mathrm{BrN}_{4} \mathrm{O}_{2} \mathrm{~S}$ & $\begin{array}{c}56.8 \\
56.81\end{array}$ & $\begin{array}{c}3.7 \\
3.77\end{array}$ & $\begin{array}{c}11.0 \\
11.04\end{array}$ \\
\hline IV a-2 & 85 & 248 & $\mathrm{C}_{24} \mathrm{H}_{20} \mathrm{~N}_{4} \mathrm{O}_{3} \mathrm{~S}$ & $\begin{array}{l}64.84 \\
64.85\end{array}$ & $\begin{array}{l}4.45 \\
4.54\end{array}$ & $\begin{array}{c}12.58 \\
12.6\end{array}$ \\
\hline IV a-3 & 84 & 298 & $\mathrm{C}_{26} \mathrm{H}_{25} \mathrm{~N}_{5} \mathrm{O}_{2} \mathrm{~S}$ & $\begin{array}{l}66.21 \\
66.22\end{array}$ & $\begin{array}{l}5.31 \\
5.34\end{array}$ & $\begin{array}{l}14.91 \\
14.85\end{array}$ \\
\hline IV b-1 & 95 & 267 & $\mathrm{C}_{24} \mathrm{H}_{19} \mathrm{BrN}_{4} \mathrm{O}_{2} \mathrm{~S}$ & $\begin{array}{l}56.82 \\
56.81\end{array}$ & $\begin{array}{l}3.72 \\
3.77\end{array}$ & $\begin{array}{l}11.08 \\
11.04\end{array}$ \\
\hline IV b-2 & 86 & 251 & $\mathrm{C}_{24} \mathrm{H}_{20} \mathrm{~N}_{4} \mathrm{O}_{3} \mathrm{~S}$ & $\begin{array}{l}64.84 \\
64.85\end{array}$ & $\begin{array}{l}4.60 \\
4.54\end{array}$ & $\begin{array}{c}12.61 \\
12.6\end{array}$ \\
\hline IV b-3 & 74 & 236 & $\mathrm{C}_{26} \mathrm{H}_{25} \mathrm{~N}_{5} \mathrm{O}_{2} \mathrm{~S}$ & $\begin{array}{l}66.24 \\
66.22\end{array}$ & $\begin{array}{l}5.34 \\
5.34\end{array}$ & $\begin{array}{l}14.92 \\
14.85\end{array}$ \\
\hline IV c-1 & 85 & 214 & $\mathrm{C}_{24} \mathrm{H}_{19} \mathrm{BrN}_{4} \mathrm{O}_{2} \mathrm{~S}$ & $\begin{array}{l}56.83 \\
56.81\end{array}$ & $\begin{array}{l}3.72 \\
3.77\end{array}$ & $\begin{array}{l}11.10 \\
11.04\end{array}$ \\
\hline IV c-2 & 96 & 268 & $\mathrm{C}_{24} \mathrm{H}_{20} \mathrm{~N}_{4} \mathrm{O}_{3} \mathrm{~S}$ & $\begin{array}{l}64.83 \\
64.85\end{array}$ & $\begin{array}{l}4.51 \\
4.54\end{array}$ & $\begin{array}{c}12.58 \\
12.6\end{array}$ \\
\hline IV c-3 & 89 & 298 & $\mathrm{C}_{26} \mathrm{H}_{25} \mathrm{~N}_{5} \mathrm{O}_{2} \mathrm{~S}$ & $\begin{array}{l}66.23 \\
66.22\end{array}$ & $\begin{array}{l}5.36 \\
5.34\end{array}$ & $\begin{array}{l}14.91 \\
14.85\end{array}$ \\
\hline
\end{tabular}




\begin{tabular}{|c|c|c|c|c|c|c|}
\hline Compound & $\begin{array}{l}\text { Yield } \\
\%\end{array}$ & $\underset{{ }^{\circ} \mathrm{C}}{\mathrm{M} . \mathrm{P}}$ & Mol. Formula & $\begin{array}{c}\text { Carbon \%, } \\
\text { (found Calcd) }\end{array}$ & $\begin{array}{l}\text { Hydrogen \%, } \\
\text { (found Calcd) }\end{array}$ & $\begin{array}{l}\text { Nitrogen \%, } \\
\text { (found Calcd) }\end{array}$ \\
\hline IV d-1 & 87 & 302 & $\mathrm{C}_{23} \mathrm{H}_{16} \mathrm{BrClN}_{4} \mathrm{O}_{2} \mathrm{~S}$ & $\begin{array}{c}52.3 \\
52.36\end{array}$ & $\begin{array}{c}3.0 \\
3.06\end{array}$ & $\begin{array}{l}10.56 \\
10.61\end{array}$ \\
\hline IV d-2 & 85 & 300 & $\mathrm{C}_{23} \mathrm{H}_{17} \mathrm{ClN}_{4} \mathrm{O}_{3} \mathrm{~S}$ & $\begin{array}{c}60.0 \\
59.42\end{array}$ & $\begin{array}{c}3.7 \\
3.69\end{array}$ & $\begin{array}{c}12.0 \\
12.03\end{array}$ \\
\hline IV d-3 & 86 & 298 & $\mathrm{C}_{25} \mathrm{H}_{22} \mathrm{ClN}_{5} \mathrm{O}_{2} \mathrm{~S}$ & $\begin{array}{c}61.0 \\
61.03\end{array}$ & $\begin{array}{c}4.5 \\
4.51\end{array}$ & $\begin{array}{c}14.3 \\
14.23\end{array}$ \\
\hline IV e-1 & 89 & 248 & $\mathrm{C}_{23} \mathrm{H}_{16} \mathrm{BrClN}_{4} \mathrm{O}_{2} \mathrm{~S}$ & $\begin{array}{c}52.3 \\
52.36\end{array}$ & $\begin{array}{c}3.0 \\
3.06\end{array}$ & $\begin{array}{l}10.56 \\
10.61\end{array}$ \\
\hline IV e-2 & 84 & 269 & $\mathrm{C}_{23} \mathrm{H}_{17} \mathrm{ClN}_{4} \mathrm{O}_{3} \mathrm{~S}$ & $\begin{array}{c}60.0 \\
59.42\end{array}$ & $\begin{array}{c}3.7 \\
3.69\end{array}$ & $\begin{array}{c}12.0 \\
12.03\end{array}$ \\
\hline IV e-3 & 87 & 289 & $\mathrm{C}_{25} \mathrm{H}_{22} \mathrm{ClN}_{5} \mathrm{O}_{2} \mathrm{~S}$ & $\begin{array}{c}61.0 \\
61.03\end{array}$ & $\begin{array}{c}4.5 \\
4.51\end{array}$ & $\begin{array}{c}14.3 \\
14.23\end{array}$ \\
\hline IV f-1 & 86 & 278 & $\mathrm{C}_{24} \mathrm{H}_{19} \mathrm{BrN}_{4} \mathrm{O}_{3} \mathrm{~S}$ & $\begin{array}{c}55.0 \\
55.07\end{array}$ & $\begin{array}{c}3.7 \\
3.66\end{array}$ & $\begin{array}{l}10.65 \\
10.70\end{array}$ \\
\hline IV f-2 & 82 & 289 & $\mathrm{C}_{24} \mathrm{H}_{20} \mathrm{~N}_{4} \mathrm{O}_{4} \mathrm{~S}$ & $\begin{array}{c}62.7 \\
62.60\end{array}$ & $\begin{array}{c}4.4 \\
4.38\end{array}$ & $\begin{array}{c}12.2 \\
12.17\end{array}$ \\
\hline IV f-3 & 81 & 269 & $\mathrm{C}_{26} \mathrm{H}_{25} \mathrm{~N}_{5} \mathrm{O}_{3} \mathrm{~S}$ & $\begin{array}{c}64.0 \\
64.05\end{array}$ & $\begin{array}{c}5.2 \\
5.17\end{array}$ & $\begin{array}{c}14.4 \\
14.36\end{array}$ \\
\hline IV g-1 & 74 & 255 & $\mathrm{C}_{24} \mathrm{H}_{19} \mathrm{BrN}_{4} \mathrm{O}_{3} \mathrm{~S}$ & $\begin{array}{l}55.08 \\
55.07\end{array}$ & $\begin{array}{l}3.70 \\
3.66\end{array}$ & $\begin{array}{l}10.69 \\
10.70\end{array}$ \\
\hline IV g-2 & 79 & 294 & $\mathrm{C}_{24} \mathrm{H}_{20} \mathrm{~N}_{4} \mathrm{O}_{4} \mathrm{~S}$ & $\begin{array}{l}62.62 \\
62.60\end{array}$ & $\begin{array}{l}4.34 \\
4.38\end{array}$ & $\begin{array}{l}12.12 \\
12.17\end{array}$ \\
\hline IV g-3 & 95 & 266 & $\mathrm{C}_{26} \mathrm{H}_{25} \mathrm{~N}_{5} \mathrm{O}_{3} \mathrm{~S}$ & $\begin{array}{l}64.10 \\
64.05\end{array}$ & $\begin{array}{l}5.21 \\
5.17\end{array}$ & $\begin{array}{l}14.34 \\
14.36\end{array}$ \\
\hline
\end{tabular}

(4-Oxo-3-o-tolyl-3,4-dihydro-quinazolin-2-ylsulfanyl)-acetic acid [1-(2-hydroxyphenyl)-ethyledene]-hydrazide ( $V a-2)$

0.1 mole of III a and 0.1 mole of 1-(2-hydroxy phenyl) acetone, $5 \mathrm{~mL}$ of DMF and few drops of acetic acid were smoothly mixed and placed inside a Pyrex-glass Erlenmeyer flask loosely corked with cotton. The mixture was irradiated with microwaves $(900 \mathrm{~W}$ $/ 2450 \mathrm{MHz}$ frequency) for 8 minutes on $50 \%$ power using a domestic microwave oven “ Sharp R-758B'. When the irradiation was stopped, the mixture was added to ice-water mixture. The solid separated was filtered, conveniently dried and recrystallized of ethanol to get $\mathrm{V}$ a-2. Yield $88 \%$, M.P. $358^{\circ} \mathrm{C}$., Mol.Formula : $\mathrm{C}_{25} \mathrm{H}_{22} \mathrm{~N}_{4} \mathrm{O}_{3} \mathrm{~S}$, Elemental analysis: Carbon, 67.8(67.85\%); Hydrogen,5.0(5.01\%); Nitrogen,12.7(12.66\%), IR (KBr): vmax, 1720(cyclic amido), 1680(acyclic >C=O), $1610 \mathrm{~cm}^{-1} .(\mathrm{C}=\mathrm{N}), \quad$ PMR(DMSO$\left.\mathrm{d}_{6}\right): \delta, 2.1\left(3 \mathrm{H}, \mathrm{s},=\mathrm{CCH}_{3}\right), 2.9\left(3 \mathrm{H}, \mathrm{s}, \mathrm{Ar}-\mathrm{CH}_{3}\right), \quad 3.74\left(2 \mathrm{H}, \quad \mathrm{s}, \mathrm{SCH}_{2}\right), \quad 7.3-8.3(12 \mathrm{H}, \mathrm{m}, \mathrm{Ar}-\mathrm{H})$, 9.6(1H,s,CONH $), 12.5(1 \mathrm{H}, \mathrm{s}, \mathrm{br}, \mathrm{OH}) \mathrm{ppm}$. 
Table 6. Spectral Characteristics

\begin{tabular}{|c|c|c|}
\hline Comp. & IR $v \max , \mathrm{cm}^{-1}$ & PMR $\delta$, ppm. \\
\hline IV b-3 & $\begin{array}{c}1720 \text { (cyclic amido), } 1680 \text { (acyclic } \\
>\mathrm{C}=\mathrm{O}), 1620(>\mathrm{C}=\mathrm{N})\end{array}$ & $\begin{array}{c}2.5\left(3 \mathrm{H}, \mathrm{s}, \mathrm{Ar}-\mathrm{CH}_{3}\right), 3.15\left(6 \mathrm{H}, \mathrm{s}, \mathrm{N}\left(\mathrm{CH}_{3}\right)_{2}\right) \\
3.73\left(2 \mathrm{H}, \mathrm{s}, \mathrm{SCH}_{2}\right), 7.2-8.2(12 \mathrm{H}, \mathrm{m}, \mathrm{Ar}-\mathrm{H}), \\
\text { 8.5(1H,s, }=\mathrm{C}-\mathrm{CH}), 9.5(1 \mathrm{H}, \mathrm{s}, \mathrm{CONH}),\end{array}$ \\
\hline IV c-3 & $\begin{array}{c}1720 \text { (cyclic amido), } 1680 \text { (acyclic } \\
>\mathrm{C}=\mathrm{O}), 1620(>\mathrm{C}=\mathrm{N})\end{array}$ & $\begin{array}{c}2.9\left(3 \mathrm{H}, \mathrm{s}, \mathrm{Ar}-\mathrm{CH}_{3}\right), 3.15\left(6 \mathrm{H}, \mathrm{s}, \mathrm{N}\left(\mathrm{CH}_{3}\right)_{2}\right) \\
3.76\left(2 \mathrm{H}, \mathrm{s}, \mathrm{SCH}_{2}\right), 7.5-8.5(12 \mathrm{H}, \mathrm{m}, \mathrm{Ar}-\mathrm{H}), \\
\quad 8.8(1 \mathrm{H}, \mathrm{s},=\mathrm{C}-\mathrm{CH}), 9.8(1 \mathrm{H}, \mathrm{s}, \mathrm{CONH}),\end{array}$ \\
\hline IV d-2 & $\begin{array}{c}3320(\text { phenolic } \mathrm{OH}) \\
1720(\text { cyclic amido }), 1680(\text { acyclic } \\
>\mathrm{C}=\mathrm{O}), 1620(>\mathrm{C}=\mathrm{N})\end{array}$ & $\begin{array}{c}3.72\left(2 \mathrm{H}, \mathrm{s}, \mathrm{SCH}_{2}\right), 7.3-8.3(12 \mathrm{H}, \mathrm{m}, \mathrm{Ar}-\mathrm{H}) \\
\text { 8.6(1H,s, }=\mathrm{C}-\mathrm{CH}), 9.5(1 \mathrm{H}, \mathrm{s}, \mathrm{CONH}) \\
12.5(1 \mathrm{H}, \mathrm{s}, \text { Broad, OH }) .\end{array}$ \\
\hline IV g-1 & $\begin{array}{l}1720 \text { (cyclic amido), } 1680 \text { (acyclic } \\
>\mathrm{C}=\mathrm{O}), 1620(>\mathrm{C}=\mathrm{N}), 1600(-\mathrm{O}-)\end{array}$ & $\begin{array}{c}3.72\left(2 \mathrm{H}, \mathrm{s}, \mathrm{SCH}_{2}\right), 3.77\left(3 \mathrm{H}, \mathrm{s}, \mathrm{OCH}_{3}\right), \\
\text { 7.3-8.3(12H,m, Ar-H),8.6(1H,s, =C-CH }), \\
9.5(1 \mathrm{H}, \mathrm{s}, \mathrm{CONH}),\end{array}$ \\
\hline
\end{tabular}

Similarly, (4-Oxo-3-o-tolyl-3,4-dihydro-quinazolin-2-ylsulfanyl)-acetic acid (1-phenyl)ethyledene]-hydrazide(V a-1), (4-Oxo-3-m-tolyl-3,4-dihydro-quinazolin-2-ylsulfanyl)-acetic acid (1-phenyl)-ethyledene]-hydrazide(V b-1), (4-Oxo-3-p-tolyl-3,4-dihydro-quinazolin-2-ylsulfanyl)acetic acid (1-phenyl)-ethyledene]-hydrazide(Vc-1), (4-Oxo-3-m-tolyl-3,4-dihydro-quinazolin-2ylsulfanyl)-acetic acid [1-(2-hydroxy-phenyl)-ethyledene]-hydrazide(V b-2), (4-Oxo-3-p-tolyl-3,4dihydro-quinazolin-2-ylsulfanyl)-acetic acid [1-(2-hydroxy-phenyl)-ethyledene]-hydrazide(V c-2), (4-Oxo-3-o-tolyl-3,4-dihydro-quinazolin-2-ylsulfanyl)-acetic acid [1-(4-methoxy-phenyl)ethyledene]-hydrazide(Va-3), (4-Oxo-3-m-tolyl-3,4-dihydro-quinazolin-2-ylsulfanyl)-acetic acid [1-(4-methoxy-phenyl)-ethyledene]-hydrazide(Vb-3), (4-Oxo-3-p-tolyl-3,4-dihydro-quinazolin-2ylsulfanyl)-acetic acid [1-(4-methoxy-phenyl)-ethyledene]-hydrazide(Vc-3), (4-Oxo-3-o-tolyl-3,4dihydro-quinazolin-2-ylsulfanyl)-acetic acid [1-(4-hydroxy-3-methoxy-phenyl)-ethyledene]hydrazide(Va-4), (4-Oxo-3-m-tolyl-3,4-dihydro-quinazolin-2-ylsulfanyl)-acetic acid [1-(4hydroxy-3-methoxy-phenyl)-ethyledene]-hydrazide( $\mathrm{Vb}-4)$, (4-Oxo-3-p-tolyl-3,4-dihydroquinazolin-2-ylsulfanyl)-acetic acid [1-(4-hydroxy-3-methoxy-phenyl)-ethyledene]-hydrazide(V c4),[3-(3-Chloro-phenyl)-4-oxo-3,4-dihydro-quinazolin-2-ylsulfanyl]-acetic acid(1-phenylethyledene)-hydrazide(Vd-1), [3-(4-Chloro-phenyl)-4-oxo-3,4-dihydro-quinazolin-2-ylsulfanyl]acetic acid(1-phenyl-ethyledene)-hydrazide(Ve-1), [3-(3-Chloro-phenyl)-4-oxo-3,4-dihydroquinazolin-2-ylsulfanyl]-acetic acid[1-(2-hydroxy-phenyl)-ethyledene)-hydrazide(Vd-2), [3-(4Chloro-phenyl)-4-oxo-3,4-dihydro-quinazolin-2-ylsulfanyl]-acetic acid[1-(2-hydroxy-phenyl)ethyledene)-hydrazide(Ve-2), [3-(3-Chloro-phenyl)-4-oxo-3,4-dihydro-quinazolin-2-ylsulfanyl]acetic acid[1-(4-methoxy -phenyl)-ethyledene)-hydrazide(Vd-3), [3-(4-Chloro-phenyl)-4-oxo-3,4dihydro-quinazolin-2-ylsulfanyl]-acetic acid[1-(4-methoxy -phenyl)-ethyledene)-hydrazide(Ve-3), [3-(3-Chloro-phenyl)-4-oxo-3,4-dihydro-quinazolin-2-ylsulfanyl]-acetic acid[1-(4-hydroxy-3methoxy-phenyl-ethyledene)-hydrazide(Vd-4), [3-(4-Chloro-phenyl)-4-oxo-3,4-dihydroquinazolin-2-ylsulfanyl]-acetic acid[1-(4-hydroxy-3-methoxy-phenyl-ethyledene)-hydrazide(Ve4), [3-(3-Methoxy -phenyl)-4-oxo-3,4-dihydro-quinazolin-2-ylsulfanyl]-acetic acid(1-phenylethyledene)-hydrazide(V f-1), [3-(4-Methoxy -phenyl)-4-oxo-3,4-dihydro-quinazolin-2ylsulfanyl]-acetic acid(1-phenyl-ethyledene)-hydrazide(V g-1), 
[3-(3- Methoxy -phenyl)-4-oxo-3,4-dihydro-quinazolin-2-ylsulfanyl]-acetic acid[1(2-hydroxy-phenyl)-ethyledene)-hydrazide(Vf-2), [3-(4- Methoxy -phenyl)-4-oxo-3,4dihydro-quinazolin-2-ylsulfanyl]-acetic acid[1-(2-hydroxy-phenyl)-ethyledene)hydrazide(Vg-2), [3-(3- Methoxy -phenyl)-4-oxo-3,4-dihydro-quinazolin-2-ylsulfanyl]acetic acid[1-(4-methoxy -phenyl)-ethyledene)-hydrazide(Vf-3), [3-(4- Methoxy phenyl)-4-oxo-3,4-dihydro-quinazolin-2-ylsulfanyl]-acetic acid[1-(4-methoxy -phenyl)ethyledene)-hydrazide(Vg-3), [3-(3- Methoxy -phenyl)-4-oxo-3,4-dihydro-quinazolin-2ylsulfanyl]-acetic acid[1-(4-hydroxy-3-methoxy-phenyl-ethyledene)-hydrazide(Vf-4) and [3-(4- Methoxy -phenyl)-4-oxo-3,4-dihydro-quinazolin-2-ylsulfanyl]-acetic acid[1(4-hydroxy-3-methoxy-phenyl-ethyledene)-hydrazide(Vg-4) were synthesised and studied for their elemental analysis and characteristic properties and subjected to PASS for prediction of biological activities.

Table 7. General characteristics and elemental analysis data of the compounds V (a-c)(1-4)

\begin{tabular}{|c|c|c|c|c|c|c|}
\hline Compound & $\begin{array}{c}\text { Yield } \\
\%\end{array}$ & $\underset{{ }^{\circ} \mathrm{C}}{\text { M.P. }}$ & Mol. Formula & $\begin{array}{c}\text { Carbon } \\
\% \text {, (found } \\
\text { Calcd) }\end{array}$ & $\begin{array}{c}\text { Hydrogen } \\
\% \text {, (found } \\
\text { Calcd) }\end{array}$ & $\begin{array}{c}\text { Nitrogen } \\
\%, \text { (found } \\
\text { Calcd) }\end{array}$ \\
\hline V a-1 & 86 & 289 & $\mathrm{C}_{25} \mathrm{H}_{22} \mathrm{~N}_{4} \mathrm{O}_{2} \mathrm{~S}$ & $\begin{array}{c}67.8 \\
67.85\end{array}$ & $\begin{array}{c}5.0 \\
5.01\end{array}$ & $\begin{array}{c}12.7 \\
12.66\end{array}$ \\
\hline $\mathrm{V}$ a-2 & 88 & 358 & $\mathrm{C}_{25} \mathrm{H}_{22} \mathrm{~N}_{4} \mathrm{O}_{3} \mathrm{~S}$ & $\begin{array}{c}65.5 \\
65.49\end{array}$ & $\begin{array}{c}4.8 \\
4.84\end{array}$ & $\begin{array}{c}12.3 \\
12.22\end{array}$ \\
\hline V a-3 & 76 & 269 & $\mathrm{C}_{26} \mathrm{H}_{24} \mathrm{~N}_{4} \mathrm{O}_{3} \mathrm{~S}$ & $\begin{array}{c}66.0 \\
66.08\end{array}$ & $\begin{array}{c}5.1 \\
5.12\end{array}$ & $\begin{array}{c}11.9 \\
11.86\end{array}$ \\
\hline V a-4 & 79 & 298 & $\mathrm{C}_{26} \mathrm{H}_{24} \mathrm{~N}_{4} \mathrm{O}_{4} \mathrm{~S}$ & $\begin{array}{c}63.9 \\
63.92\end{array}$ & $\begin{array}{l}4.99 \\
4.95\end{array}$ & $\begin{array}{c}11.5 \\
11.47\end{array}$ \\
\hline V b-1 & 85 & 325 & $\mathrm{C}_{25} \mathrm{H}_{22} \mathrm{~N}_{4} \mathrm{O}_{2} \mathrm{~S}$ & $\begin{array}{c}67.8 \\
67.85\end{array}$ & $\begin{array}{c}5.0 \\
5.01\end{array}$ & $\begin{array}{c}12.7 \\
12.66\end{array}$ \\
\hline $\mathrm{V} b-2$ & 74 & 321 & $\mathrm{C}_{25} \mathrm{H}_{22} \mathrm{~N}_{4} \mathrm{O}_{3} \mathrm{~S}$ & $\begin{array}{c}65.5 \\
65.49\end{array}$ & $\begin{array}{c}4.8 \\
4.84\end{array}$ & $\begin{array}{c}12.3 \\
12.22\end{array}$ \\
\hline $\mathrm{V} b-3$ & 89 & 314 & $\mathrm{C}_{26} \mathrm{H}_{24} \mathrm{~N}_{4} \mathrm{O}_{3} \mathrm{~S}$ & $\begin{array}{c}66.0 \\
66.08\end{array}$ & $\begin{array}{c}5.1 \\
5.12\end{array}$ & $\begin{array}{c}11.9 \\
11.86\end{array}$ \\
\hline V b-4 & 96 & 322 & $\mathrm{C}_{26} \mathrm{H}_{24} \mathrm{~N}_{4} \mathrm{O}_{4} \mathrm{~S}$ & $\begin{array}{c}63.8 \\
63.92\end{array}$ & $\begin{array}{l}4.92 \\
4.95\end{array}$ & $\begin{array}{l}11.48 \\
11.47\end{array}$ \\
\hline V c-1 & 85 & 301 & $\mathrm{C}_{25} \mathrm{H}_{22} \mathrm{~N}_{4} \mathrm{O}_{2} \mathrm{~S}$ & $\begin{array}{c}67.8 \\
67.85\end{array}$ & $\begin{array}{c}5.0 \\
5.01\end{array}$ & $\begin{array}{c}12.7 \\
12.66\end{array}$ \\
\hline $\mathrm{V} \mathrm{c}-2$ & 84 & 289 & $\mathrm{C}_{25} \mathrm{H}_{22} \mathrm{~N}_{4} \mathrm{O}_{3} \mathrm{~S}$ & $\begin{array}{c}65.5 \\
65.49\end{array}$ & $\begin{array}{c}4.8 \\
4.84\end{array}$ & $\begin{array}{c}12.3 \\
12.22\end{array}$ \\
\hline $\mathrm{V} \mathrm{c}-3$ & 88 & 279 & $\mathrm{C}_{26} \mathrm{H}_{24} \mathrm{~N}_{4} \mathrm{O}_{3} \mathrm{~S}$ & $\begin{array}{c}66.0 \\
66.08\end{array}$ & $\begin{array}{c}5.1 \\
5.12\end{array}$ & $\begin{array}{c}11.9 \\
11.86\end{array}$ \\
\hline V c-4 & 75 & 299 & $\mathrm{C}_{26} \mathrm{H}_{24} \mathrm{~N}_{4} \mathrm{O}_{4} \mathrm{~S}$ & $\begin{array}{l}63.88 \\
63.92\end{array}$ & $\begin{array}{l}4.96 \\
4.95\end{array}$ & $\begin{array}{l}11.45 \\
11.47\end{array}$ \\
\hline
\end{tabular}


Table 8.General characteristics and elemental analysis data of the compounds V (d,e)(1-4)

\begin{tabular}{ccccccc}
\hline Compound & $\begin{array}{c}\text { Yield } \\
\%\end{array}$ & $\begin{array}{c}\text { M.P. } \\
{ }^{\circ} \mathrm{C}\end{array}$ & Mol. Formula & $\begin{array}{c}\text { Carbon } \\
\text {, (found } \\
\text { Calcd) }\end{array}$ & $\begin{array}{c}\text { Hydrogen } \\
\text {, (found } \\
\text { Calcd) }\end{array}$ & $\begin{array}{c}\text { Nitrogen } \\
\% \text {, (found } \\
\text { Calcd) }\end{array}$ \\
\hline V d-1 & 71 & 300 & $\mathrm{C}_{24} \mathrm{H}_{19} \mathrm{ClN}_{4} \mathrm{O}_{2} \mathrm{~S}$ & 62.2 & 4.1 & 12.2 \\
& & & & 62.27 & 4.14 & 12.10 \\
V d-2 & 75 & 302 & $\mathrm{C}_{24} \mathrm{H}_{19} \mathrm{ClN}_{4} \mathrm{O}_{3} \mathrm{~S}$ & 60.10 & 4.10 & 11.80 \\
& & & & 60.19 & 4.00 & 11.70 \\
V d-3 & 85 & 305 & $\mathrm{C}_{25} \mathrm{H}_{21} \mathrm{ClN}_{4} \mathrm{O}_{3} \mathrm{~S}$ & 61.00 & 4.30 & 11.40 \\
& & & & 60.91 & 4.29 & 11.36 \\
V d-4 & 86 & 325 & $\mathrm{C}_{25} \mathrm{H}_{21} \mathrm{ClN}_{4} \mathrm{O}_{4} \mathrm{~S}$ & 58.9 & 4.20 & 11.00 \\
& & & & 59.0 & 4.16 & 11.01 \\
V e-1 & 86 & 321 & $\mathrm{C}_{24} \mathrm{H}_{19} \mathrm{ClN}_{4} \mathrm{O}_{2} \mathrm{~S}$ & 62.2 & 4.10 & 12.2 \\
& & & & 62.27 & 4.14 & 12.10 \\
V e-2 & 82 & 341 & $\mathrm{C}_{24} \mathrm{H}_{19} \mathrm{ClN}_{4} \mathrm{O}_{3} \mathrm{~S}$ & 60.15 & 4.10 & 11.81 \\
& & & & 60.19 & 4.00 & 11.70 \\
V e-3 & 83 & 289 & $\mathrm{C}_{25} \mathrm{H}_{21} \mathrm{ClN}_{4} \mathrm{O}_{3} \mathrm{~S}$ & 61.10 & 4.21 & 11.35 \\
& & & & 60.91 & 4.29 & 11.36 \\
V e-4 & 91 & 257 & $\mathrm{C}_{25} \mathrm{H}_{21} \mathrm{ClN}_{4} \mathrm{O}_{4} \mathrm{~S}$ & 58.89 & 4.21 & 11.10 \\
& & & & 59.0 & 4.16 & 11.01 \\
\hline
\end{tabular}

Table 9.General characteristics and elemental analysis data of the compounds V (f,g)(1-4)

\begin{tabular}{|c|c|c|c|c|c|c|}
\hline Compound & $\begin{array}{l}\text { Yield } \\
\%\end{array}$ & $\underset{{ }^{\circ} \mathrm{C}}{\mathrm{M} . P .}$ & $\begin{array}{l}\text { Mol. } \\
\text { Formula }\end{array}$ & $\begin{array}{l}\text { Carbon } \\
\% \text { (found } \\
\text { calcd) }\end{array}$ & $\begin{array}{c}\text { Hydrogen } \\
\%(\text { found } \\
\text { calcd })\end{array}$ & $\begin{array}{c}\text { Nitrogen } \\
\% \text { (found } \\
\text { calcd) }\end{array}$ \\
\hline \multirow{2}{*}{ V f-1 } & \multirow{2}{*}{72} & \multirow{2}{*}{288} & \multirow{2}{*}{$\mathrm{C}_{25} \mathrm{H}_{22} \mathrm{~N}_{4} \mathrm{O}_{3} \mathrm{~S}$} & 65.50 & 4.88 & 12.25 \\
\hline & & & & 65.49 & 4.84 & 12.22 \\
\hline \multirow{2}{*}{$\mathrm{V} \mathrm{f}-2$} & \multirow{2}{*}{85} & \multirow{2}{*}{259} & \multirow{2}{*}{$\mathrm{C}_{25} \mathrm{H}_{22} \mathrm{~N}_{4} \mathrm{O}_{4} \mathrm{~S}$} & 63.23 & 4.70 & 11.78 \\
\hline & & & & 63.28 & 4.67 & 11.81 \\
\hline \multirow{2}{*}{$\mathrm{V} \mathrm{f}-3$} & \multirow{2}{*}{90} & \multirow{2}{*}{222} & \multirow{2}{*}{$\mathrm{C}_{26} \mathrm{H}_{24} \mathrm{~N}_{4} \mathrm{O}_{3} \mathrm{~S}$} & 64.02 & 5.00 & 11.51 \\
\hline & & & & 63.92 & 4.95 & 11.47 \\
\hline \multirow[t]{2}{*}{$\mathrm{V} \mathrm{f}-4$} & \multirow{2}{*}{88} & \multirow{2}{*}{269} & \multirow{2}{*}{$\mathrm{C}_{26} \mathrm{H}_{24} \mathrm{~N}_{4} \mathrm{O}_{4} \mathrm{~S}$} & 61.88 & 4.72 & 11.00 \\
\hline & & & & 61.89 & 4.79 & 11.10 \\
\hline \multirow[b]{2}{*}{ V g-1 } & \multirow{2}{*}{74} & \multirow{2}{*}{299} & \multirow{2}{*}{$\mathrm{C}_{25} \mathrm{H}_{22} \mathrm{~N}_{4} \mathrm{O}_{3} \mathrm{~S}$} & 65.51 & 4.89 & 12.23 \\
\hline & & & & 65.49 & 4.84 & 12.22 \\
\hline \multirow{2}{*}{ V g-2 } & \multirow{2}{*}{71} & \multirow{2}{*}{278} & \multirow{2}{*}{$\mathrm{C}_{25} \mathrm{H}_{22} \mathrm{~N}_{4} \mathrm{O}_{4} \mathrm{~S}$} & 63.33 & 4.67 & 11.82 \\
\hline & & & & 63.28 & 4.67 & 11.81 \\
\hline \multirow{2}{*}{ V g-3 } & \multirow{2}{*}{73} & \multirow{2}{*}{274} & \multirow{2}{*}{$\mathrm{C}_{26} \mathrm{H}_{24} \mathrm{~N}_{4} \mathrm{O}_{3} \mathrm{~S}$} & 64.11 & 5.10 & 11.45 \\
\hline & & & & 63.92 & 4.95 & 11.47 \\
\hline \multirow{2}{*}{ V g-4 } & \multirow{2}{*}{82} & \multirow{2}{*}{275} & \multirow{2}{*}{$\mathrm{C}_{26} \mathrm{H}_{24} \mathrm{~N}_{4} \mathrm{O}_{4} \mathrm{~S}$} & 61.91 & 4.78 & 11.20 \\
\hline & & & & 61.89 & 4.79 & 11.10 \\
\hline
\end{tabular}


Table 10. Spectral Characteristics

\begin{tabular}{|c|c|c|}
\hline Comp. & IR $v \max , \mathrm{cm}^{-1}$ & PMR $\delta, \quad p p m$. \\
\hline V a-1 & $\begin{array}{l}\text { 1720(cyclic amido), 1680(acyclic } \\
>\mathrm{C}=\mathrm{O}), 1620(>\mathrm{C}=\mathrm{N})\end{array}$ & $\begin{array}{l}\therefore 1\left(3 \mathrm{H}, \mathrm{s},=\mathrm{CCH}_{3}\right), 2.5\left(3 \mathrm{H}, \mathrm{s}, \mathrm{Ar}-\mathrm{CH}_{3}\right), \\
\left.\text { i.73(2H,s, } \mathrm{SCH}_{2}\right), 7.2-8.2(13 \mathrm{H}, \mathrm{m}, \mathrm{Ar}-\mathrm{H}), \\
1.5(1 \mathrm{H}, \mathrm{s}, \mathrm{CONH}),\end{array}$ \\
\hline V a-3 & $\begin{array}{l}\text { 1720(cyclic amido), 1680(acyclic } \\
>\mathrm{C}=\mathrm{O}), 1620(>\mathrm{C}=\mathrm{N}), 1600(-\mathrm{O}-) .\end{array}$ & $\begin{array}{l}.2\left(3 \mathrm{H}, \mathrm{s},=\mathrm{CCH}_{3}\right) 2.6\left(3 \mathrm{H}, \mathrm{s}, \mathrm{Ar}-\mathrm{CH}_{3}\right), \\
.76\left(2 \mathrm{H}, \mathrm{s}, \mathrm{SCH}_{2}\right), 7.5-8.5(12 \mathrm{H}, \mathrm{m}, \mathrm{Ar}-\mathrm{H}), \\
1.8(1 \mathrm{H}, \mathrm{s}, \mathrm{CONH}),\end{array}$ \\
\hline $\mathrm{V} \mathrm{c}-2$ & $\begin{array}{l}3310(\text { phenolic } \mathrm{OH}) \\
1720(\text { cyclic amido }), 1680(\text { acyclic } \\
>\mathrm{C}=\mathrm{O}), 1620(>\mathrm{C}=\mathrm{N})\end{array}$ & $\begin{array}{l}\therefore 1\left(3 \mathrm{H}, \mathrm{s},=\mathrm{CCH}_{3}\right), 2.8\left(3 \mathrm{H}, \mathrm{s}, \mathrm{Ar}-\mathrm{CH}_{3}\right), \\
.72\left(2 \mathrm{H}, \mathrm{s}, \mathrm{SCH}_{2}\right), 7.3-8.3(12 \mathrm{H}, \mathrm{m}, \mathrm{Ar}-\mathrm{H}), \\
1.5(1 \mathrm{H}, \mathrm{s}, \mathrm{CONH}), 12.5(1 \mathrm{H}, \mathrm{s}, \mathrm{Broad}, \mathrm{OH}) .\end{array}$ \\
\hline V e-1 & $\begin{array}{l}1720(\text { cyclic amido), } 1680 \text { (acyclic } \\
>\mathrm{C}=\mathrm{O}), 1620(>\mathrm{C}=\mathrm{N}) .\end{array}$ & 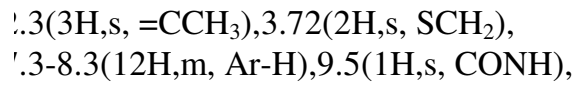 \\
\hline
\end{tabular}

Table 11.QSAR Analysis of Activities with $\mathrm{Pa}>70 \% 3$ Possible activities at $\mathrm{Pa}>70 \%$

\begin{tabular}{cccccccc}
\hline Activity & {$[1] \mathrm{Pa}$} & {$[1] \mathrm{Pi}$} & {$[2] \mathrm{Pa}$} & {$[2] \mathrm{Pi}$} & {$[3] \mathrm{Pa}$} & {$[3] \mathrm{Pi}$} & {$[4]$} \\
Compounds & & & & & & & \\
\hline IV a-1 & 0,838 & 0,004 & 0,780 & 0,004 & 0,757 & 0,005 & 48 \\
IV a-2 & 0,822 & 0,005 & 0,754 & 0,004 & 0,740 & 0,005 & 50 \\
IV a-3 & 0,818 & 0,005 & 0,746 & 0,005 & 0,746 & 0,005 & 49 \\
IV b-1 & 0,804 & 0,006 & 0,785 & 0,004 & 0,768 & 0,005 & 49 \\
IV b-2 & 0,781 & 0,007 & 0,758 & 0,004 & 0,751 & 0,005 & 51 \\
IV b-3 & 0,774 & 0,007 & 0,751 & 0,005 & 0,756 & 0,005 & 50 \\
IV c-1 & 0,809 & 0,006 & 0,792 & 0,004 & 0,775 & 0,005 & 48 \\
IV c-2 & 0,786 & 0,006 & 0,764 & 0,004 & 0,756 & 0,005 & 50 \\
IV c-3 & 0,780 & 0,007 & 0,756 & 0,004 & 0,762 & 0,005 & 49 \\
IV d-1 & 0,821 & 0,005 & 0,775 & 0,004 & 0,759 & 0,005 & 50 \\
IV d-2 & 0,802 & 0,006 & 0,751 & 0,005 & 0,744 & 0,005 & 52 \\
IV d-3 & 0,797 & 0,006 & 0,743 & 0,005 & 0,749 & 0,005 & 51 \\
IV e-1 & 0,818 & 0,005 & 0,782 & 0,004 & 0,765 & 0,005 & 49 \\
IV e-2 & 0,798 & 0,006 & 0,757 & 0,004 & 0,749 & 0,005 & 51 \\
IV e-3 & 0,793 & 0,006 & 0,749 & 0,005 & 0,754 & 0,005 & 50 \\
IV f-1 & 0,787 & 0,006 & 0,759 & 0,004 & 0,744 & 0,005 & 53 \\
IV f-2 & 0,766 & 0,007 & 0,748 & 0,005 & 0,739 & 0,005 & 53 \\
IV f-3 & 0,752 & 0,007 & 0,731 & 0,005 & 0,734 & 0,005 & 54 \\
IV g-1 & 0,792 & 0,006 & 0,764 & 0,004 & 0,748 & 0,005 & 52 \\
IV g-2 & 0,772 & 0,007 & 0,752 & 0,005 & 0,743 & 0,005 & 52 \\
IV g-3 & 0,758 & 0,007 & 0,735 & 0,005 & 0,738 & 0,005 & 53 \\
\hline
\end{tabular}

[1] HDL-cholesterol increasing [3] Antimycobacterial [2] Antituberculosic

[4] Substructure descriptors 
Table 12. QSAR Analysis of compounds V(a-g)(1-4)Activities with Pa>70\%Only 1 Possible activitiy at $\mathrm{Pa}>70 \% \mathrm{HDL}-$ cholesterol increasing Activity

\begin{tabular}{cccc}
\hline Compound & Pa & Pi & Substructure Descriptors \\
\hline V a-1 & 0.838 & 0.004 & 43 \\
V a-2 & 0.821 & 0.005 & 50 \\
V a-3 & 0.818 & 0.005 & 51 \\
V a-4 & 0.808 & 0.006 & 57 \\
V b-1 & 0.800 & 0.006 & 44 \\
V b-2 & 0.781 & 0.007 & 51 \\
V b-3 & 0.776 & 0.007 & 52 \\
V b-4 & 0.765 & 0.007 & 58 \\
V c-1 & 0.806 & 0.006 & 43 \\
V c-2 & 0.786 & 0.006 & 50 \\
V c-3 & 0.781 & 0.007 & 51 \\
V c-4 & 0.770 & 0.007 & 57 \\
V d-1 & 0.818 & 0.005 & 46 \\
V d-2 & 0.800 & 0.006 & 53 \\
V d-3 & 0.797 & 0.006 & 54 \\
V d-4 & 0.787 & 0.006 & 60 \\
V e-1 & 0.815 & 0.005 & 45 \\
V e-2 & 0.797 & 0.006 & 53 \\
V e-3 & 0.793 & 0.006 & 53 \\
V e-4 & 0.782 & 0.007 & 59 \\
V f-1 & 0.780 & 0.007 & 49 \\
V f-2 & 0.766 & 0.007 & 54 \\
V f-3 & 0.780 & 0.007 & 49 \\
V f-4 & 0.766 & 0.007 & 56 \\
V g-1 & 0.785 & 0.006 & 48 \\
V g-2 & 0.772 & 0.007 & 53 \\
V g-3 & 0.785 & 0.006 & 48 \\
V g-4 & 0.771 & 0.007 & 55 \\
\hline
\end{tabular}

\section{Conclusions}

The compounds of the type (4-Oxo-3-aryl -3,4-dihydro-quinazolin-2-ylsulfanyl)-acetic acid (substituted-benzyledene)-hydrazide IV (a-g)(1-3) were studied for the predictions of their probabilities of being active $[\mathrm{Pa}]$ and inactive [Pi] for the selected activities such that the $\mathrm{Pa}>70 \%$.A software application (PASS) was used for this purpose. The relationship between structure and different biological activities was studied. It was found that the 2-methy phenyl quinazolinones are expected to exhibit spectacular HDL Cholesterol Increasing activity, whereas the 3- chloro-phenyl and 4-chlorophenyl quinazolinone condensed with 4-bromobenzaldehyde are expected to exhibit spectacular HDL Cholesterol Increasing activity. Other derivatives are also expected to exhibit good HDL Cholesterol Increasing activity. Hence these compounds are recommended for the screening of HDL Cholesterol Increasing activity. When the relationship between the structure and anti-tuberculosic 
activity was studied, it was found that the 3-methylphenyl, 4-methyl-phenyl,3-chloro-phenyl and 4-chlorophenyl quinazolinone derivatives condensed with 4-bromobenzaldehyde are expected to exhibit spectacular anti-tuberculosic activity. Whereas, other derivatives are also expected to exhibit good anti-tuberculosic activity.Hence these compounds are recommended for the screening of anti-tuberculosic activity .

QSAR study of the compounds was similarly done for the anti-mycobacerial activities. It was found that 3-methylphenyl, 4-methyl-phenyl and 4-chloro-phenyl quinazolinone derivatives condensed with 4-bromobenzaldehyde are expected to exhibit spectacular antimycobacterial activity. Whereas, other derivatives are also expected to exhibit good antimycobacterial activity. Hence these compounds were recommended for the screening of antimycobacterial activity too.

Similarly the derivatives of quinazolinones condensed with different aromatic ketones were when subjected to PASS, we found only one activity at $\mathrm{Pa}>70 \%$. The derivatives with $\mathrm{R}_{1}=2$-Me are expected to exhibit spectacular HDL cholesterol increasing activity. Whereas the derivatives of quinazolinones condensed with unsubstituted benzophenone were found to be more probably active as compared with those with substituted ones. In fact the quinazolinones condensed with different aromatic ketones are expected to be exhibit more HDL cholesterol activity as compared to those with aldehydes.

Hence these compounds were recommended for the screening of HDL Cholesterol Increasing activity, anti-tuberculosic activity anti-mycobacterial activity too.

\section{Acknowledgement}

One of the authors, Savita Dhongade (Desai) thanks U.G.C. for sanctioning Minor Research Project.

\section{References}

1. Caddick S, Tetrahedron, 1995, 51, 10403; Varma, R. S. Green Chemistry, 1999, 43.

2. Bose A K, Banik B K, Lavlinskaia N, Jayaraman M and Manhas M S, Chemtech,1997, 27, 18.

3. Giuere R J, Namen A M, Lopez B O, Arepally A, Ramos D E, Majetich G and Defauw J. Tetrahedron Lett, 1987, 28, 6553; Berlan J, Giboreau P, Lefeuvre S and Marchand C Tetrahedron Lett, 1991,32, 2363.

4 Giuere R J, Bray T L, Duncan S M and Majetich G Tetrahedron Lett, 1986, 27, 4945.

5 Ipaktschi J and Bruck M Chem Ber, 1990,123, 1591; Srikrishna A and Nagaraju S, J Chem Soc Perkin Trans,1992, 1, 311.

6. Singh J, Sharma M, Kad G L and Chhabra B R J Chem Res(S),1997, 342 ; Mitra A K, De A and Karchaudhuri N, J Chem Res(S), 1999, 246.

7. Bose A K, Banik B K, Barakat K J and Manhas M S, Synlett, 1993, 5758; Wang C-D, Shi X-Z and Xie R-J, Synth Commun, 1997, 27, 2517; Beregszaszi T and Molnar A, Synth Commun, 1997, 27, 3705.

9. Varma R S, Chatterjee A K and Varma M, Tetrahedron Lett, 1993, 34, 3207

10 Mitra A K, De A and Karchaudhuri N, Synlett, 1998, 1345.

11. Mitra A K, De A and Karchaudhuri N, Indian J Chem, 2000, 39B,

12 Ley S V and Mynett D M, Synlett,1993, 793; Loupy A, Pigeon P,Ramadani M \& Jaequault P,Synth Commun, 1994, 24, 159. 
13. Mitra A K, De A and Karchaudhuri N, Synth Commun, 1999, 29, 573.

14 Mitra A K, De A and Karchaudhuri N, Synth Commun, 1999, 29, 2731.

15 Almena I, Carrillo J R, de la Cruz P, Díaz-Ortiz A, Gómez-Escalonilla M J, de la Hoz A, Langa F, Prieto P and Sánchez-Migallon A, Targets in Heterocyclic Systems, 1998, 2, 281.

16. Bogdal D, Pielichowski J and Jaskott K, Heterocycles, 1997, 45, 715; Bogdal D, Pielichowski J and Jaskott K, Synth. Commun., 1997, 27, 1553; Bogdal D, Molecules, 1999, 4, 333.

17. Regen S L, Czech B and Quici S, Pol. J. Chem., 1981, 55, 843.

18 Reid W and Hirsh C, J Org Chem, 1966, 33, 143.

19. Moskalenko N Yu, Yakhontov L N , Zhikharera G P, Pershin G N, Peters V V, Eustratova M I and Mulikovskaya I M, Khimfarm Zh, 1986, 20(4), 437; Chem Abstr, 1987,106, 3297v

20 Tiwari S S and Pandey M P, Acta Ciencia Indica Chem, 1990, 16c(3), 251; Chem Abstr, 1992,116, 2355557q

21. Tyagi R, Goel B, Srivastava V K and Kumar A, Indian J Pharm Sci , 1998, 60(5), 283.

22. Kumar A, Verma R S , Jaju B P and Sinha J N , J Indian Chem Soc , 1990,67, 920.

23. Kamal A and Reddy A V N , Indian J Chem, ,1985, 24B , 414.

24. Husain M L ,Srivastava G C and Dua P R , Indian J Chem, 1982,21B, 381.

25. Ottoviano G, Boll Soc Itgal Biol Sper, 1963, 39(24), 1968.

26. Gupta D P, Ahmad S, Kumar A, and Shankar K, Indian J Chem, 1988, 27B, 1060.

27 Kumar A, Gurtus, Sinha J N , Bhargava K P and Shankar, Eur J Med Chem, 1985, 20(1), 95.

28. Goodman L S and Gilman A, The Pharmacological Basis of Therapeutics , $7^{\text {th }}$ edn (CollirMacMillan , London ), 1985,B

29. http//www.ibmh.msk.su/PASS 


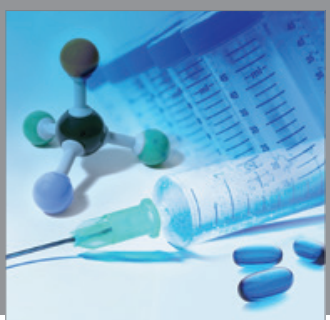

International Journal of

Medicinal Chemistry

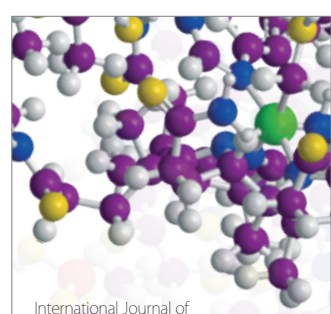

Carbohydrate Chemistry

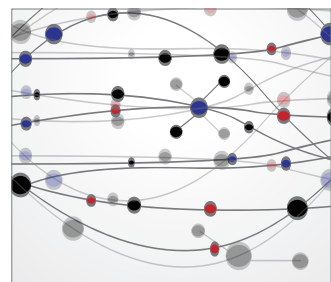

The Scientific World Journal
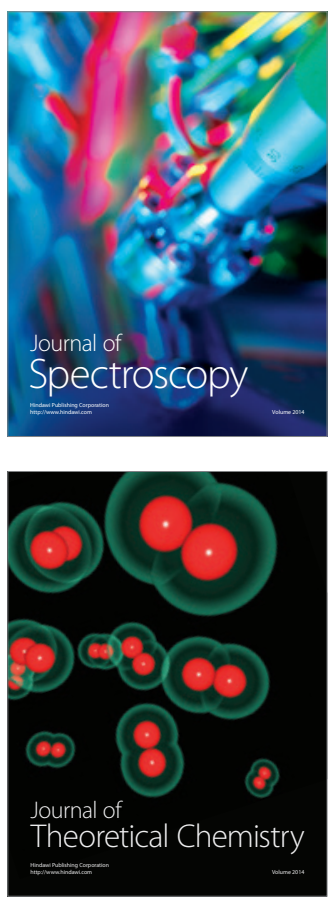
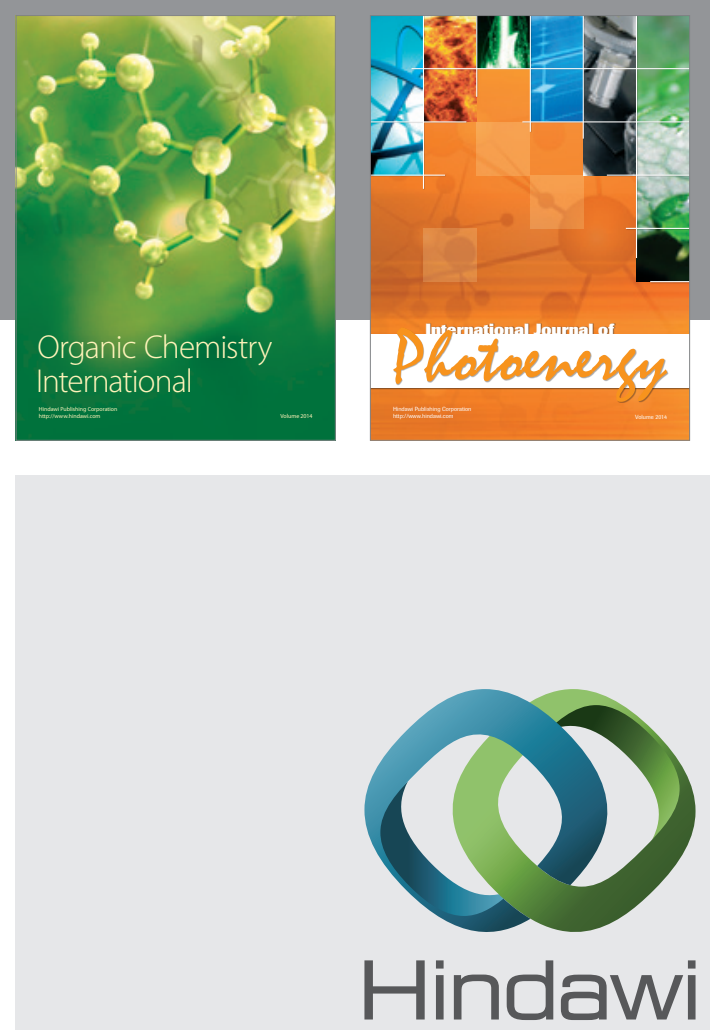

Submit your manuscripts at

http://www.hindawi.com
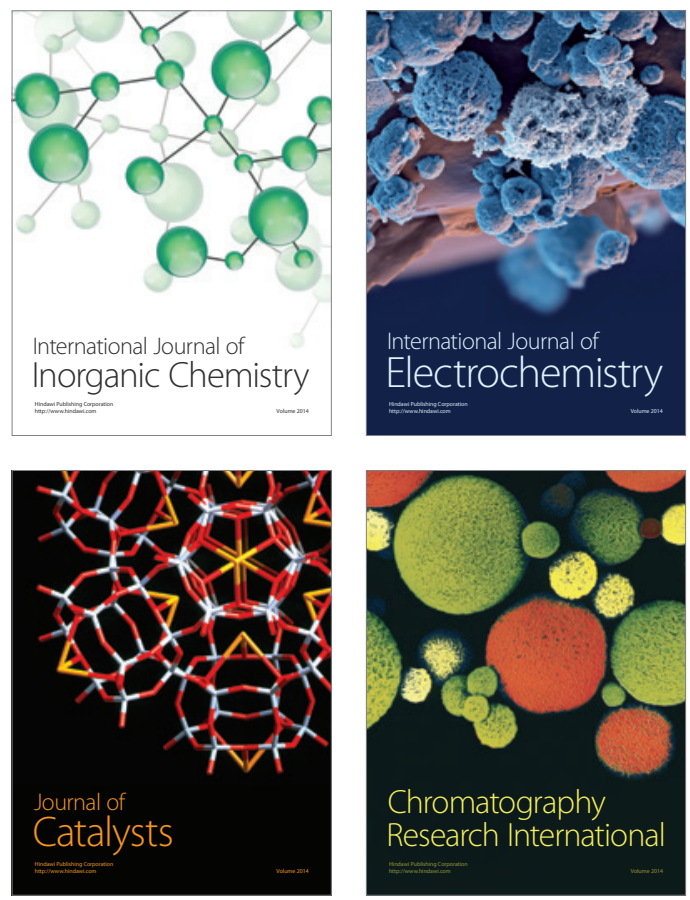
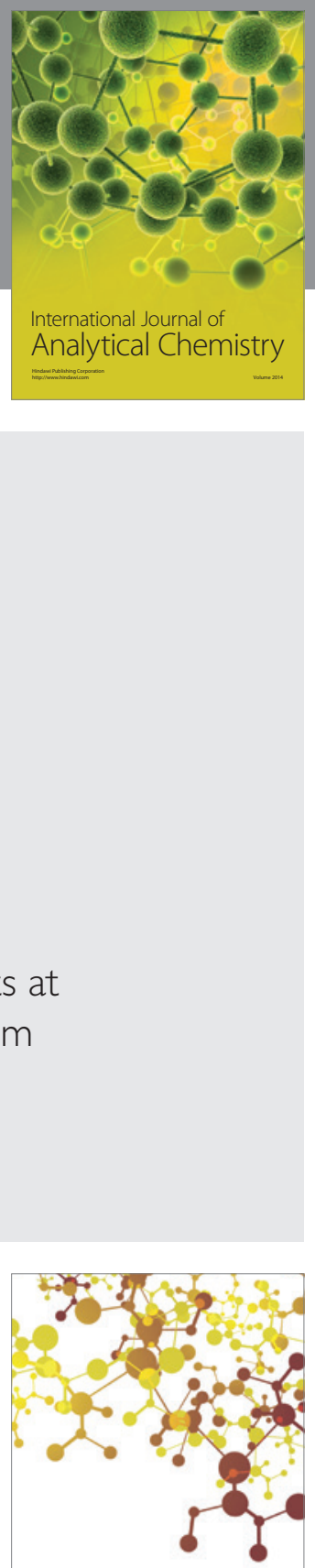

Journal of

Applied Chemistry
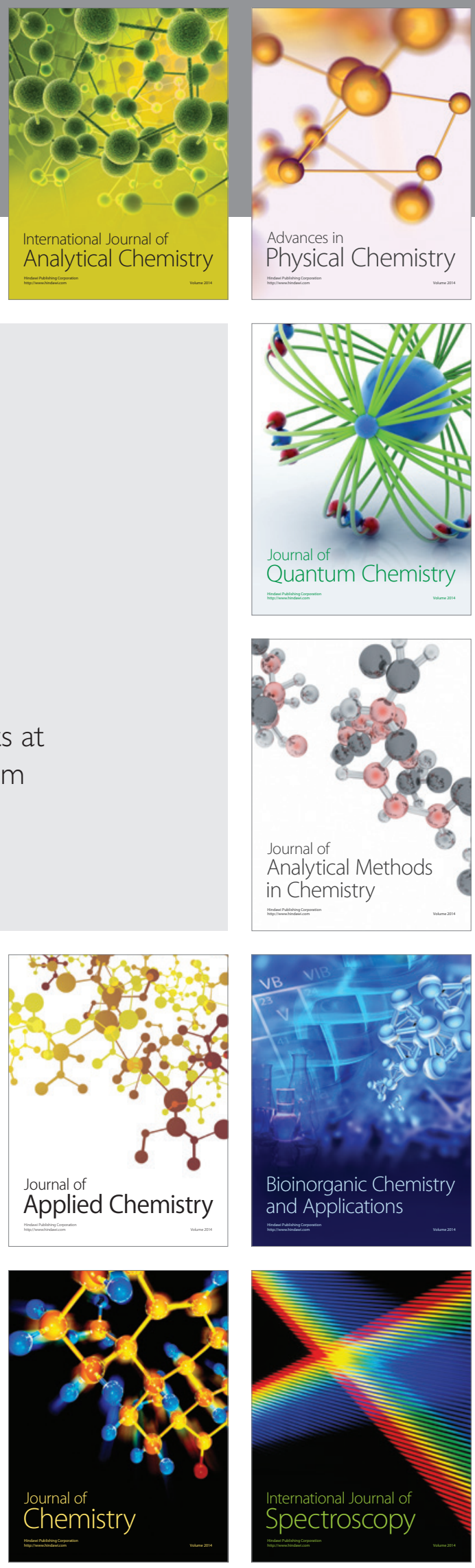